\title{
Subject Index Vol. 56, 1991
}

Acupuncture 2

AIDS 66

Anodal block 234

Basal ganglia 44 Blockade, local neural 2 Blood-brain barrier 197 Brachytherapy 135 Braintumor 135 BRW stereotactic System 193

Cerebral blood flow 197

- metabolism 197

Cerebrospinal fluid 234

Charged particles 197

Computed tomography-guided. stereotaxis

104,109, 179 Computer modeling 234

Deafferentation 166

Delayed radiation injury 197

Depth electrodes 129

Dorsal root entry zone lesions 166

Electrical Stimulation 2, 250 Epidural electrodes 77

- spinal cord Stimulation 234

Epilepsy 129, 145

Farial pain 166

Fiber size selectivity 234

Functional neurosurgery 109

Glioblastoma 135 Glycerol 159 
Headframe 193 Head injury 184

Immunogold colloid 66 Immunohistochemical staining methods

66 Instrumentation 129 Intraoperative monitoring 28 Ionizingradiation 213

Low back 2

Meckel's cave 37 Motor control 44 Multiple sclerosis 184

Neocortex 145

Nerve Aber recruitment 234

Nerves 250

Nucleus caudalis 166

Pain 2,20,77,250

Pallidotomy 109

Paresthesia threshold 234

Patil stereotactic System, intraoperative

calibration 179 Percutaneous microcompression 28, 37 Placebo 2 Efessüre monitoring 37

Radiosurgery 213 Radiotherapy 135,213 Recovery cycle 145 Rhizolysis 159 Rigidity 44 
Sacralplexus 250 Somibrace 193 Spinal cord Stimulation 20,77 StereoadapterTM 109

Stereotactic apparatus 213

- biopsy 66

- -, technique 179

- radiosurgery 197

- surgery $104,109,129,179$

- technique 109,135,184,213 Stretch reflex, primates 44 
Thalamotomy 104, 109,184 Toxoplasma gondii 66 Transcutaneous electncal Stimulation 2,

159 Tremor 184 Tngeminal evoked Potentials 28

- nerve 37

- neuralgia 28,37,159

- nucleotomy 166

Unstable angina 20 